OPEN ACCESS

Edited by:

Peter Sörös,

University of Oldenburg, Germany

Reviewed by:

Tomasz Maciej Rutkowski,

University of Tokyo, Japan

Luigi Tamè,

Birkbeck, University of London, UK

*Correspondence: Hidehiko Okamoto hokamoto@nips.ac.jp

Received: 13 May 2016 Accepted: 17 January 2017 Published: 06 February 2017

Citation:

Okamoto H and Kakigi R (2017)

Modulation of Auditory Evoked Magnetic Fields Elicited by Successive Frequency-Modulated (FM) Sweeps.

Front. Hum. Neurosci. 11:36. doi: 10.3389/fnhum.2017.00036

\section{Modulation of Auditory Evoked Magnetic Fields Elicited by Successive Frequency-Modulated (FM) Sweeps}

\author{
Hidehiko Okamoto ${ }^{1,2 *}$ and Ryusuke Kakigi ${ }^{1,2}$ \\ ${ }^{1}$ Department of Integrative Physiology, National Institute for Physiological Sciences, Okazaki, Japan, ${ }^{2}$ Department of \\ Physiological Sciences, Graduate University for Advanced Studies, Hayama, Japan
}

In our daily life, we are successively exposed to frequency-modulated (FM) sounds that play an important role in speech and species-specific communication. Previous studies demonstrated that repetitive exposure to identical pure tones resulted in decreased neural activity. However, the effects of repetitively presented FM sounds on neural activity in the human auditory cortex remain unclear. In the present study, we used magnetoencephalography to investigate auditory evoked $\mathrm{N} 1 \mathrm{~m}$ responses elicited by four successive temporally repeated and superimposed FM sweeps in three sequences: (1) four FM sweeps were identical, (2) four FM sweeps had the same FM direction and rate, but different carrier frequencies, (3) four FM sweeps differed with respect to the FM rate and/or direction and their carrier frequencies. In contrast to our expectations, the results obtained demonstrated that $\mathrm{N} 1 \mathrm{~m}$ responses were maximal when the four FM sweeps were identical and minimal when they were distinct. These results suggest that the neural processing of repetitive FM sweeps in the human auditory cortex may differ from that of repetitive pure tones.

Keywords: adaptation, auditory cortex, brain, frequency modulation (FM), habituation, human, magnetoencephalography (MEG)

\section{INTRODUCTION}

In daily life, we are continually exposed to repetitive sound signals, such as the ticking of a clock, that are irrelevant for listeners. However, these sound signals are easily ignored and neural resources are conserved for unexpected changes in the acoustic environment (Bregman, 1990). The decrements elicited in neural activity by repetitive auditory signals appear to play an important role in this process. Previous studies on humans demonstrated that the first pure tone elicits a maximal N1/ N1m response, which is a major deflection in electroencephalography, or magnetoencephalography (MEG) waveforms with a latency of approximately $0.1 \mathrm{~s}$, and subsequent tones elicit smaller N1/N1m responses (see a review, Nätänen and Picton, 1987). The decrement in neural activity elicited by repetitive stimuli is not limited to the auditory modality (Butler, 1968; Fruhstorfer et al., 1970; Budd et al., 1998; Uhlig et al., 2016), it is also observed in visual (Rugg et al., 1995; Buckner et al., 1998; Kourtzi and Kanwisher, 2000) and somatosensory modalities (Allison, 1962; Angel et al., 1985; Otsuru et al., 2011) in the human brain.

Even though adaptive phenomena may be observed over long timescales such as the evolution of a species, we focused on the neural adaptation that occurs within the timescales of hundreds of 
$\mathrm{ms}$ to seconds in the present study. Using auditory oddball paradigms, in which a high-probability sound ("standard") and low-probability sound ("deviant") were randomly presented, Ulanovsky et al. (2003) demonstrated that neurons in the cat primary auditory cortex showed stronger neural activity corresponding to "deviant" tones rather than "standard" tones because of the neural mechanism underlying stimulus-specific adaptation. Excitatory and inhibitory neural networks within the central auditory pathway appear to enhance the neural processing of stimulus-specific adaptation (Taaseh et al., 2011; Malmierca et al., 2015) and may contribute to better auditory novelty detection (Malmierca et al., 2014; Chen et al., 2015). However, most of the previous studies that investigated stimulusspecific adaptation used simple pure tones as sound stimuli; therefore, neural decrements induced by repetitive complex sound signals remain elusive.

The neural encoding of frequency modulated (FM) sweeps appears to differ, at least partially, from that of pure tones. Pure tones vibrate specific portions of the basilar membrane according to the tonotopic map in the cochlea and the vibration patterns of the basilar membrane remain constant during the pure tone presentation (Reale and Imig, 1980; Schreiner and Langner, 1988; Robles and Ruggero, 2001). In contrast, FM sweeps change their vibration patterns of the basilar membrane over time. Therefore, in order to process FM sound signals, their carrier frequencies as well as FM rates and directions need to be analyzed simultaneously (Deboer and Dreschler, 1987; Eggermont, 1998; Zatorre et al., 2002; Obleser et al., 2008). Previous studies showed that FM sweeps caused stronger neural activity than pure tones in the primary auditory cortex of marmosets (Liang et al., 2002) as well as stronger non-primary auditory area activity than that in the primary auditory cortex in cats (Tian and Rauschecker, 1994, 1998; Heil and Irvine, 1998). Moreover, a recent MEG study measuring the neural activity elicited by temporally repeated and superimposed FM sweeps revealed that lower-rate FM sweeps (1 and 4 octaves per sec) elicited larger N1m source strengths and shorter N1m latencies than higher-rate FM sweeps (16 and 64 octaves per sec; Okamoto and Kakigi, 2015). FM sweeps in human speech play an essential role in verbal communications. For example, changing the FM direction of the third formant within a human voiced "ba" sound turned speech perception into "ga" (Liberman et al., 1957). In order to follow speech, it is inevitable for humans to execute the proper neural processing of FM sweeps that repeatedly appear in daily conversations; however, the neural responses elicited by repeatedly presented FM sweeps remain elusive in the human auditory cortex.

The aim of the present study was to investigate the adaptation of auditory evoked $\mathrm{N} 1 \mathrm{~m}$ responses elicited by four successive FM sweeps using MEG. We used temporally repeated and superimposed FM sweeps that were matched in the spectral domain, but differed in their direction and modulation rate as adaptor stimuli (AS) and test stimuli (TS) (Figure 1) and presented them using an adaptation paradigm, in which trains of four successive FM sweeps were presented in three manners: (1) AS and three consequent TS were completely identical ("Identical" sequence), (2) AS and TS had the same FM direction and rate, but different carrier frequencies ("Category" sequence),
(3) AS and TS differed with respect to the FM rate and/or direction and their carrier frequencies ("Distinct" sequence). Crucially, we used overall (i.e., in sum) identical AS and TS between sequences, and thereby controlled neural activity differences caused by different FM sweeps. Similar to the N1m responses elicited by successive pure tones, we hypothesized that the auditory evoked N1m responses eliciting the successive FM sweeps may be the smallest in the "Identical" sequence and the largest in the "Distinct" sequence.

\section{MATERIALS AND METHODS}

\section{Participants}

Thirteen healthy people (nine females; mean \pm standard deviation: $25.7 \pm 7.4$ years) participated in the present study. All participants were right handed [assessed via Edinburgh Handedness Inventory (Oldfield, 1971)] and had no history of otological or neurological disorders. They were fully informed about the study and gave written informed consent for their participation. The study was approved by the Ethics Commission of the National Institute for Physiological Sciences and conformed to The Code of the World Medical Association (Declaration of Helsinki).

\section{Stimuli and Experimental Design}

The experimental design is schematically displayed in Figure $\mathbf{1 .}$ Adaptor stimuli (AS) were followed by three test stimuli (TS). AS and TS had a duration of $0.25 \mathrm{~s}$ with 0.01 -s linear onset and offset ramps (sampling rate: $48,000 \mathrm{~Hz}$ ). They were temporally repeated and superimposed FM sweeps similar to those used in our previous study (Okamoto and Kakigi, 2015). They consisted of six FM tones that traversed an upward or downward direction within the $500-2000 \mathrm{~Hz}$ frequency range with a modulation rate of 4 or 16 octaves per sec, resulting in four FM sweeps (FM_up_04, FM_up_16, FM_down_04, and FM_down_16). FM_up_04 and FM_up_16 had 25- and 6.25-ms linear rise ramps starting at 500 $\mathrm{Hz}$ and 25- and 6.25-ms linear fall ramps ending at $2000 \mathrm{~Hz}$, whereas FM_down_04 and FM_down_16 had 25- and 6.25-ms linear rise ramps starting at $2000 \mathrm{~Hz}$ and $25-$ and $6.25-\mathrm{ms}$ linear fall ramps ending at $500 \mathrm{~Hz}$, respectively (Figure 1). The rise and fall ramps of the FM tones simultaneously started in order to minimize the sound envelope change.

We prepared 48 FM sweeps that were characterized by different initial spectral components in each FM type (FM_up_04, FM_up_16, FM_down_04, or FM_down_16), resulting in 192 AS and TS. We presented AS and three successive TS (TS_1, TS_2, and TS_3) with an inter-stimulus interval of $0.5 \mathrm{~s}$ in specific manners (Figure 1). In the "Identical" sequence, four identical sounds were successively presented (Audio 1 in Supplementary Material). In the "Category" sequence, AS and TS belonging to the same FM type (FM_up_04, FM_up_16, FM_down_04, or FM_down_16), but differing with respect to carrier frequencies were presented (Audio 2 in Supplementary Material). In the "Distinct" sequence, four distinct FM types with different carrier frequencies were successively presented (Audio 3 in Supplementary Material). Each FM sweep appeared only once at a given position (AS, TS_1, TS_2, or TS_3) in each sequence 


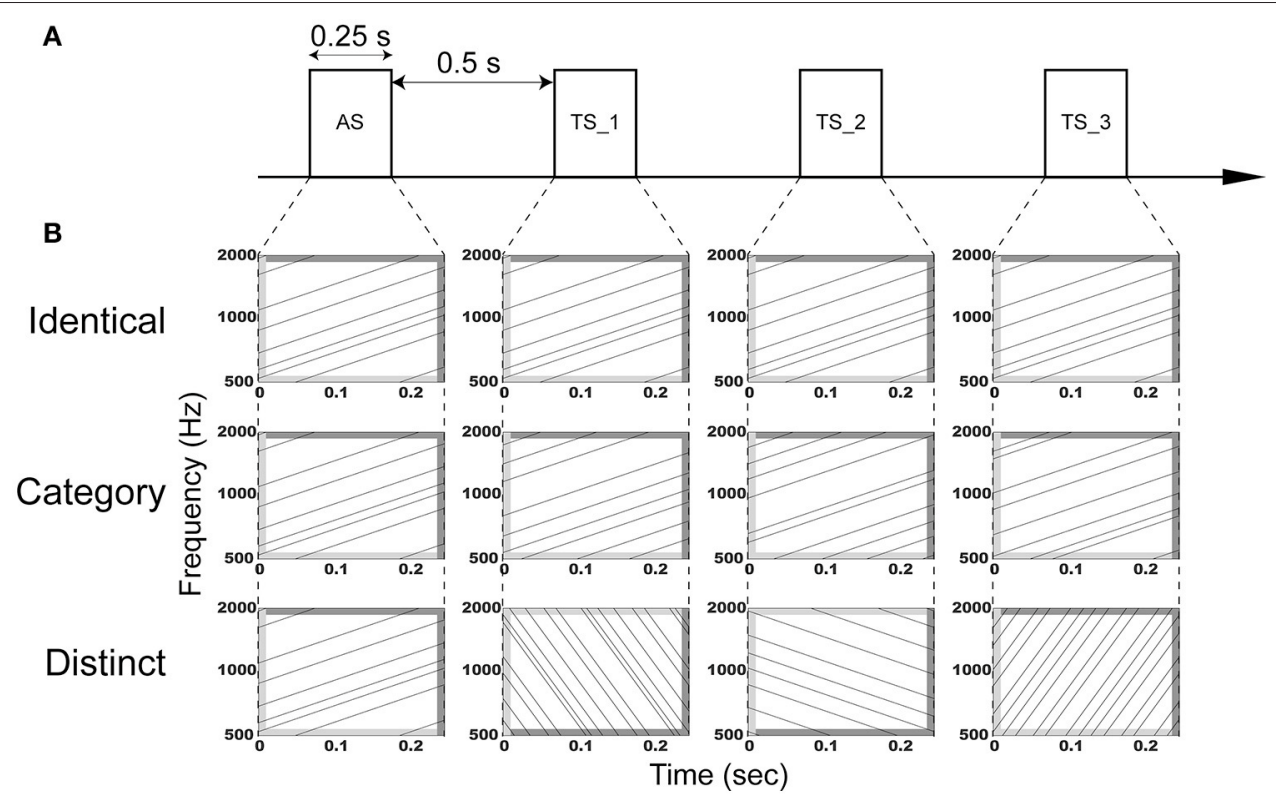

FIGURE 1 | Schematic display of the auditory stimulation. (A) Adaptor stimuli (AS) and subsequent test stimuli (TS_1, TS_2, and TS_3) with a duration of 0.25 s were presented with an inter-stimulus interval of $0.5 \mathrm{~s}$. (B) AS and TS consisted of six temporally repeating frequency-modulated (FM) tones that traversed an upward or downward direction with a modulation rate of 4 or 16 octaves per sec. The dark and light gray areas represent the linear rise- and fall-ramps of the sound signals, respectively. In the "Identical" sequence (upper row), AS, TS_1, TS_2, and TS_3 were identical. In the "Category" sequence (middle row), AS, TS_1, TS_2, and TS_3 were characterized by the same modulation rate and direction, but different carrier frequencies. In the "Distinct" sequence (lower row), AS, TS_1, TS_2, and TS_3 had a distinct modulation rate and/or direction. Exemplary sound files of "Identical," "Category," and "Distinct" sequences are available online as Audio 1, Audio 2, and Audio 3 in Supplementary Material, respectively.

("Identical," "Category," or "Distinct"). Therefore, total sound inputs were counter-balanced between positions and between sequences. The "Identical," "Category," and "Distinct" sequences were pseudo-randomly presented. The silent interval between the preceding TS_3 and the subsequent AS was $2.5 \mathrm{~s}$.

All FM sweeps were adjusted to have equal energy and presented by insert earphones (E-ARTONE 3A, Aearo Company Auditory Systems, Indianapolis, IN) through $1.5-\mathrm{m}$ plastic tubes attached to foam plugs (E-A-RLINK, Aearo Company Auditory Systems, Indianapolis, IN). Prior to starting the MEG experiment, we examined each participant's hearing threshold for FM_up_04 in each ear. During the MEG recording session, TS were diotically presented at an intensity of $50 \mathrm{~dB}$ more than the individual hearing threshold. Participants were comfortably seated upright and were instructed to watch a self-chosen silent movie with captions in order to keep them alert during the MEG measurement.

\section{Data Acquisition and Analysis}

Auditory evoked magnetic fields were recorded with a helmetshaped 204-channel whole head planar-type gradiometer (Vector-view, ELEKTA, Neuromag, Helsinki, Finland) located in a magnetically shielded and acoustically quiet room. Prior to the MEG recording, four head position indicator coils were attached to the participant's scalp. A 3D digitizer (Polhemus Inc., Colchester, VT) was used to measure the locations of head position indicator coils and three anatomical landmarks, nasion, and bilateral pre-auricular points, and head shapes. A current was fed to the four head position indicator coils and the resulting magnetic fields were used to assess the head position of the participant with respect to the MEG dewar. Signals were filtered online using a bandpass of $0.1-200-\mathrm{Hz}$ and digitized at 1000 $\mathrm{Hz}$. The magnetic fields starting $0.15 \mathrm{~s}$ prior to the sound onset and ending $0.15 \mathrm{~s}$ after the sound offset were averaged selectively for each position (AS, TS_1, TS_2, and TS_3) in each sequence ("Identical," "Category," or "Distinct") irrespective of the FM types (FM_up_04, FM_up_16, FM_down_04, or FM_down_16). Epochs containing amplitude values of $>3 \mathrm{pT} / \mathrm{cm}$ were discarded as artifact-contaminated epochs.

In order to analyze auditory evoked $\mathrm{N} 1 \mathrm{~m}$ responses, we estimated N1m source locations and orientations by means of two single equivalent current dipoles (one for each hemisphere) using the brain electric source analysis software package (BESA Research 5.3.7, BESA GmbH, Germany). In the N1m source analysis, the grand-averaged magnetic field signals elicited by all FM sweeps after artifact rejection were $30 \mathrm{~Hz}$ low-pass filtered (zero-phase shift Butterworth filter, $24 \mathrm{~dB} / \mathrm{oct}$ ), and the baseline was corrected relative to the 0.1 -s pre-stimulus interval. The peak $\mathrm{N} 1 \mathrm{~m}$ response was initially identified as the maximal rootmean square value of the global field power of all the sensors within the time interval from 0.075 to $0.15 \mathrm{~s}$ after the test stimulus onset. The single equivalent current dipole model was used for source locations and orientations based on the 0.01$\mathrm{s}$ time window around the $\mathrm{N} 1 \mathrm{~m}$ peak using all the sensors for each participant and hemisphere. The locations and orientations of the equivalent current dipoles corresponding to the $\mathrm{N} 1 \mathrm{~m}$ 
responses were individually determined in a Cartesian coordinate system with the medial-lateral axis connecting the pre-auricular points, the posterior-anterior axis passing through the nasion perpendicular to the medial-lateral axis, and the inferiorsuperior axis orthogonal to the medial-lateral and posteriorlateral axes. The estimated N1m source location and orientation were used to calculate the source strength waveform as a spatial filter in each participant and hemisphere (Tesche et al., 1995). Thereafter, we obtained the maximal N1m source strengths and $\mathrm{N} 1 \mathrm{~m}$ latencies in each sequence and each position based on the calculated source strength waveforms.

In order to examine hemispheric differences in the $\mathrm{N} 1 \mathrm{~m}$ source strengths elicited by FM sweeps, we calculated the mean N1m source strengths elicited by AS and TS in each hemisphere and each participant. We then performed planned comparisons (paired two-tailed $t$-tests) between the left and right hemispheres. Thereafter, in order to avoid the source strength difference between participants and hemispheres, the source strengths of the N1m responses elicited by TS_1, TS_2, and TS_3 were individually normalized with respect to the N1m source strength elicited by AS in each sequence and each hemisphere. Normalized N1m source strengths and N1m latencies were evaluated by means of threeway repeated-measures analysis of variances (ANOVAs) using Hemisphere (Left vs. Right), Sequence ("Identical," "Category," and "Distinct"), and Position (TS_1, TS_2, and TS_3) as factors. Thereafter, Bonferroni-corrected paired $t$-tests were performed for post hoc multi-comparisons. Statistical analyses were performed using SPSS (V 21, IBM Corp.). We applied the Shapiro-Wilk test to establish whether data were normally distributed.

\section{RESULTS}

It was possible to average a sufficient number of trials for each condition in 13 participants after artifact rejection (mean \pm standard deviation: $189.8 \pm 3.0$ ), and clear auditory evoked N1m responses were obtained under each condition (cf. Figure 2). The goodness-of-fit of the underlying dipolar source models for the averaged MEG waveforms of all the

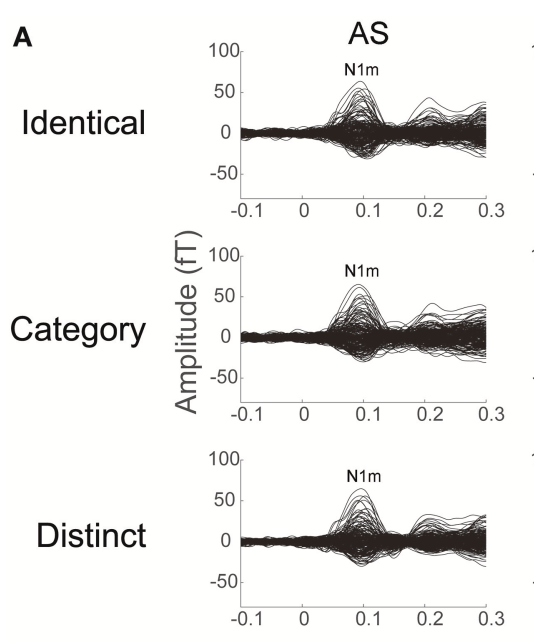

B
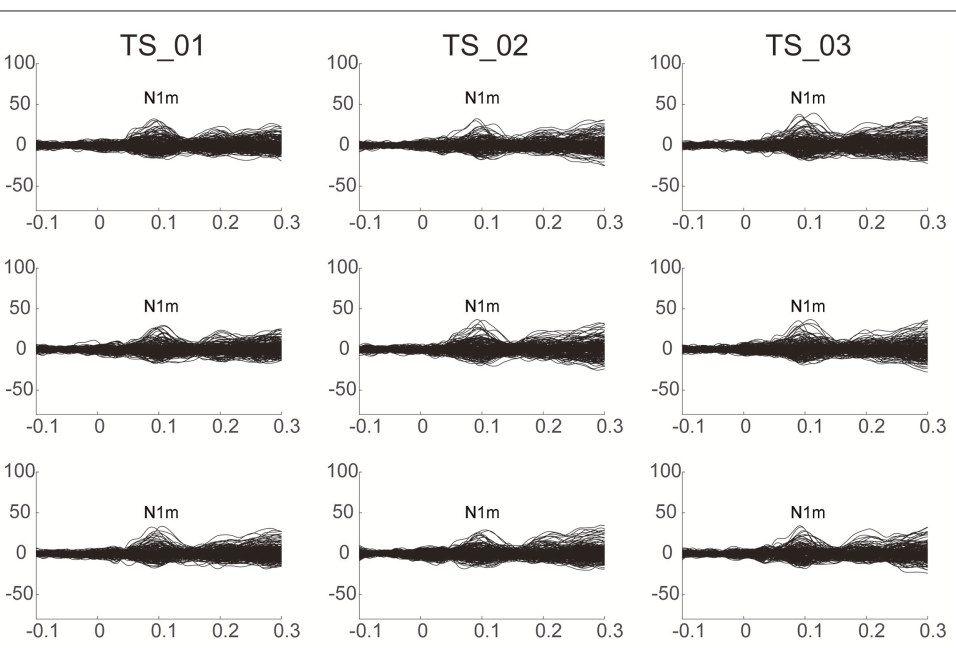

Latency (sec)
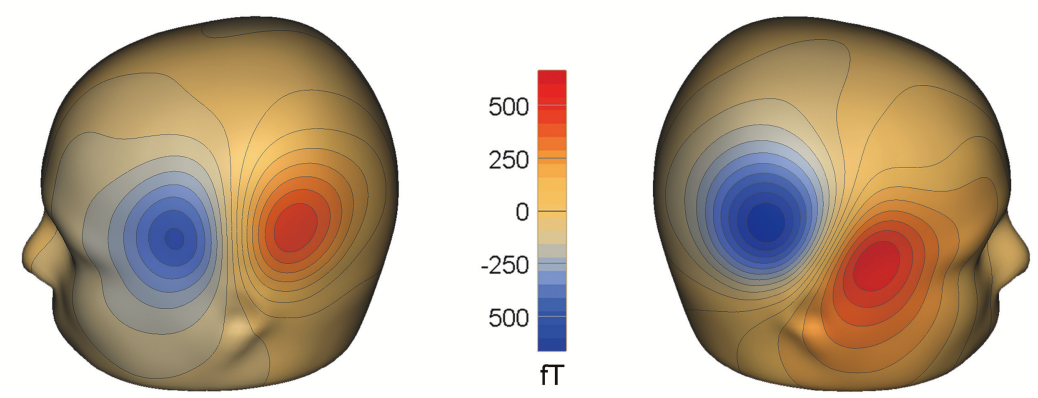

FIGURE 2 | Examples of individual magnetic waveforms. (A) Each column represents the auditory evoked fields elicited by the adaptor stimuli (AS) and three test stimuli (TS_1, TS_2, and TS_3) (from left to right) in the "Identical" (upper row), "Category" (middle row), and "Distinct" (lower row) sequences, respectively. (B) Iso-contour maps of the magnetic fields at the N1m latency of the grand-averaged magnetic waveforms across AS, TS_1, TS_2, and TS_3. The magnetic contour maps show clear dipolar patterns above the left (left panel) and right (right panel) hemispheres. Red and blue contour lines represent the outbound and inbound flows of magnetic fields from and into the brain. 
gradiometers was more than $90 \%$ in all participants (mean \pm standard deviation: $96.4 \pm 1.8 \%)$.

The time courses of the N1m source strengths (from -0.1 to $+0.3 \mathrm{~s})$ grand-averaged across all participants $(N=13)$ are displayed in Figure 3. An N1m response with a latency of approximately $0.1 \mathrm{~s}$ is clearly shown. According to the ShapiroWilk test, data were normally distributed using the mean N1m source strengths elicited by AS and TS in each hemisphere as a dependent variable (Left Hemisphere: $p=0.515$; Right Hemisphere: $p=0.599$ ). The planned comparison applied to the $\mathrm{N} 1 \mathrm{~m}$ source strength revealed a significant difference between hemispheres $\left[t_{(1,12)}=2.53, p=0.027\right]$. N1m responses had larger source strengths in the right than in the left hemisphere.

Figure 4 shows mean normalized N1m source strengths in each sequence ("Identical," "Category," or "Distinct"), in each hemisphere (Left or Right), and in each position (AS, TS_1, TS_2, or TS_3) together with the corresponding 95\% confidence intervals obtained by boot-strap resampling tests (iteration $=100,000)$. According to the Shapiro-Wilk test, data were normally distributed using the mean normalized $\mathrm{N} 1 \mathrm{~m}$ source strengths in each sequence, hemisphere, and position as a dependent variable ("Identical_Left_TS_1": $p=0.87$; "Identical_Left_TS_2": $p=0.91$; “Identical_Left_TS_3": $p=$ 0.26 ; “Identical_Right_TS_1": $p=0.97$; “Identical_Right_TS_2": $p=0.22$; "Identical_Right_TS_3": $p=0.16$; “Category_Left_ TS_1": $p=0.18$; "Category_Left_TS_2": $p=0.83$; "Category_
Left_TS_3": $p=0.69$; “Category_Right_TS_1": $p=0.17$; "Category_Right_TS_2": $p=0.23$; “Category_Right_TS_3": $p=$ 0.27 ; “Distinct_Left_TS_1": $p=0.42$; “Distinct_Left_TS_2": $p$ $=0.67$; “Distinct_Left_TS_3": $p=0.40$; “Distinct_Right_TS_1": $p=0.63$; “Distinct_Right_TS_2": $p=0.91$; “Distinct_Right_ TS_3": $p=0.38)$. The three-way repeated-measures ANOVA applied to normalized N1m source strengths revealed significant main effects for Sequence $\left[F_{(2,24)}=11.26, p<0.001\right]$ and Hemisphere $\left[F_{(1,13)}=4.74, p=0.05\right]$, and a significant interaction between Sequence and Hemisphere $\left[F_{(2,24)}=6.87, p\right.$ $=0.004]$; however, no significant main effect for Position $\left[F_{(2,24)}\right.$ $=1.38, p=0.27]$ was found. The Bonferroni-corrected post hoc multi-comparisons revealed significant differences between "Identical" and "Category" $\left.\left[t_{(12}\right)=3.09, p=0.028\right]$ and between "Identical" and "Distinct" $\left[t_{(12)}=4.22, p=0.004\right]$; however, no significant difference between "Category" and "Distinct" $\left[t_{(12)}=\right.$ 2.27, $p=0.127$ ] was found, as shown in Figure 5 .

Figure 6 shows the mean N1m latencies in each hemisphere (Left or Right), in each sequence ("Identical," "Category," or "Distinct"), and in each position (AS, TS_1, TS_2, or TS_3) together with the corresponding 95\% confidence intervals obtained by boot-strap resampling tests (iteration $=100,000)$. According to the Shapiro-Wilk test, data were normally distributed using the $\mathrm{N} 1 \mathrm{~m}$ latencies in each sequence, hemisphere, and position as a dependent variable ("Identical_Left_TS_1": $p=0.991$; “Identical_Left_TS_2":

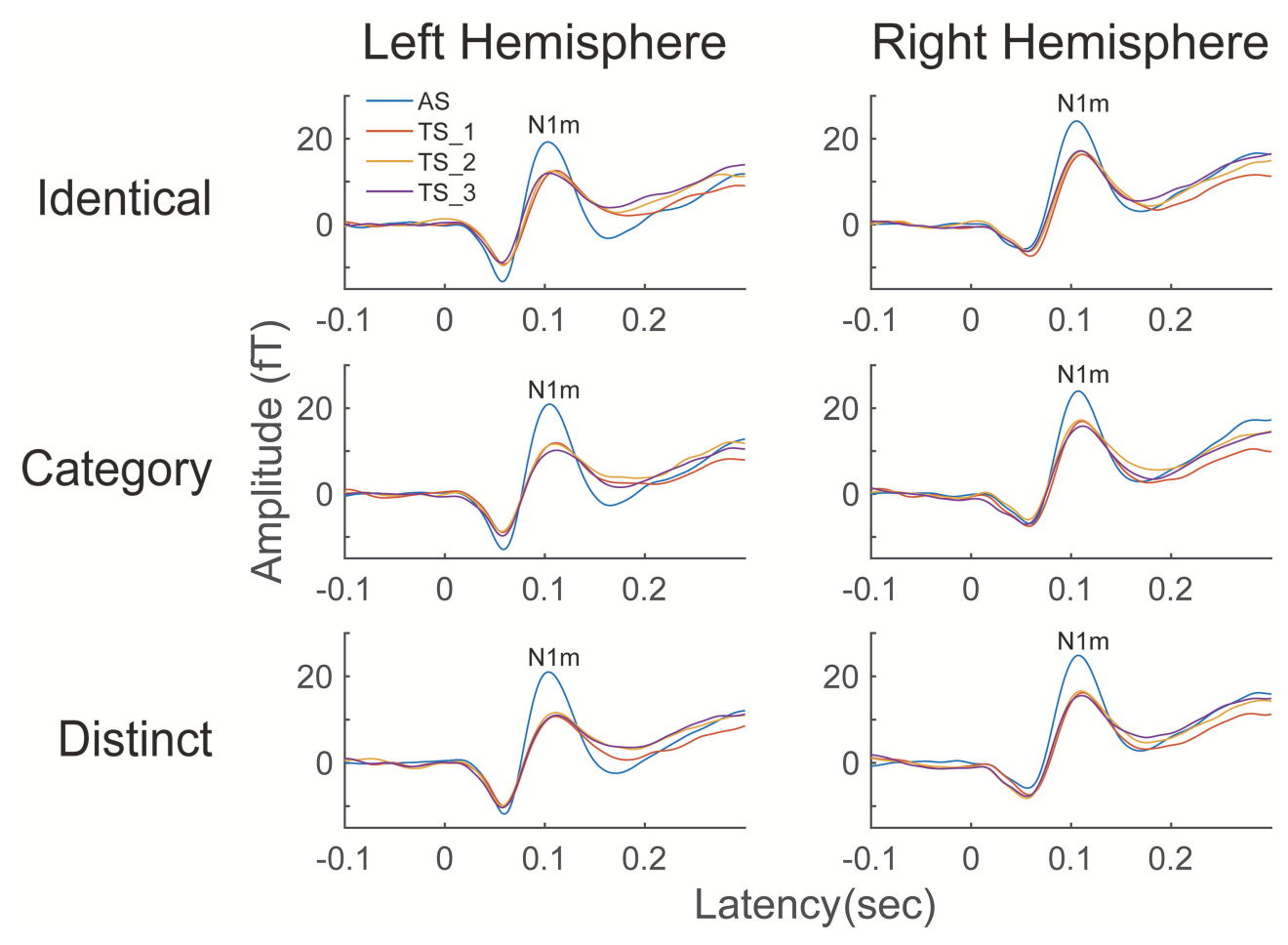

FIGURE 3 | Source strength waveforms calculated at the N1m generator averaged across 13 participants in the left and right hemispheres. Top, middle, and bottom rows represent the source strength waveforms in the "Identical," "Category," and "Distinct" sequences, respectively. Each colored line indicates the position of the test stimuli (TS): The 1st (AS), 2nd (TS_1), 3rd (TS_2), and 4th (TS_3) FM sweeps were colored as blue, red, yellow, and purple, respectively (see legends in the left upper corner). 

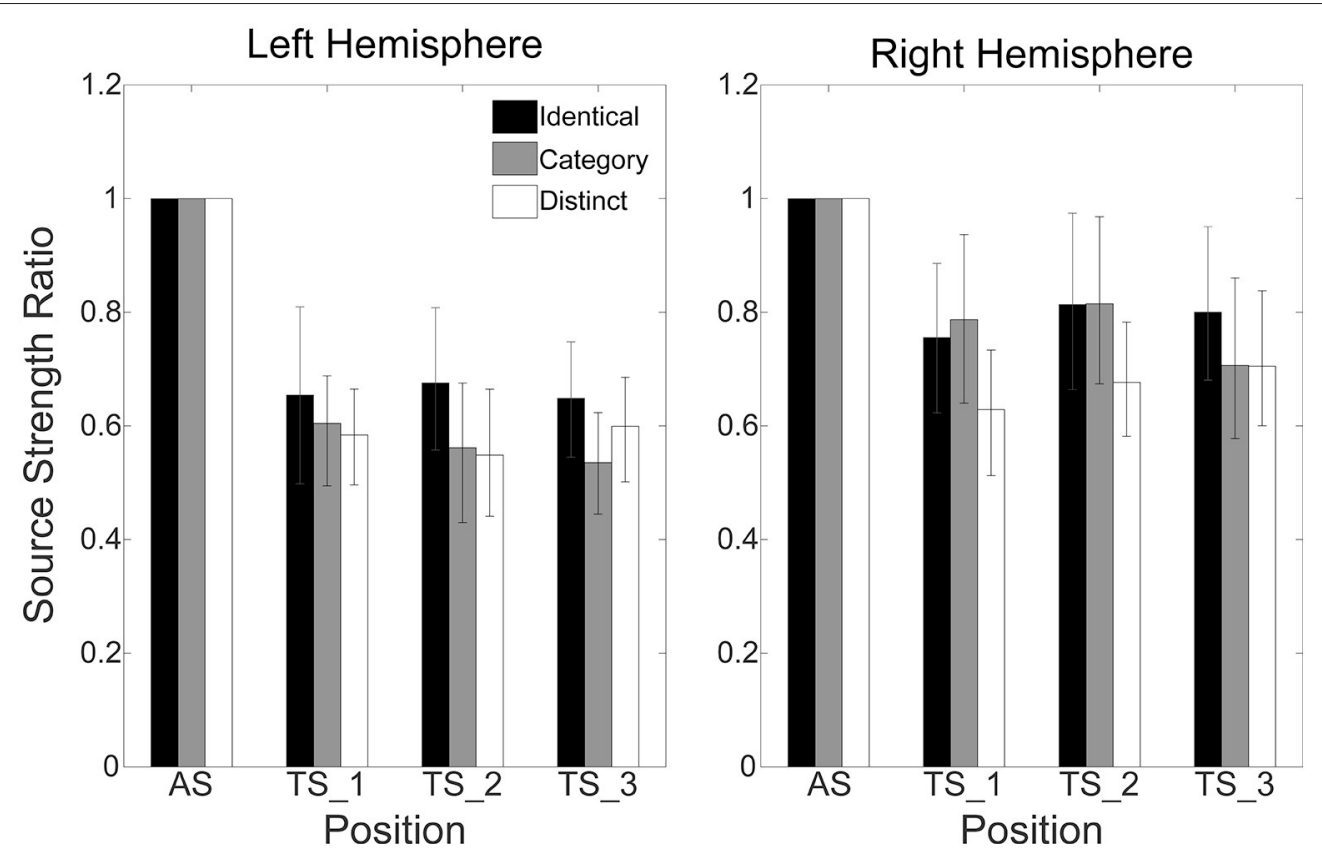

FIGURE 4 | Group means $(N=13)$ of normalized N1m source strengths in the left (left panel) and right (right panel) hemispheres elicited by the 1st (AS), 2nd (TS_1), 3rd (TS_2), and 4th (TS_3) trains of four successive FM sweeps, including error bars denoting 95\% confidence intervals. Black, gray, and white bars denote the "Identical," "Category," and "Distinct" sequences, respectively.

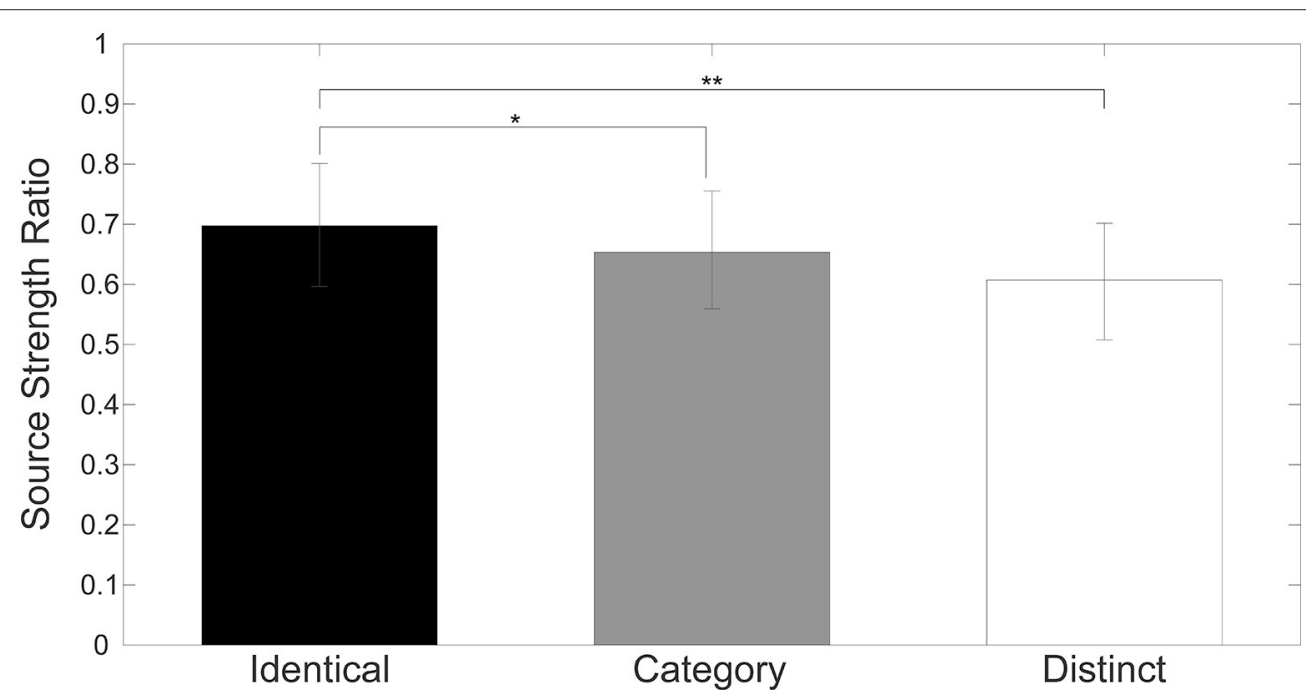

FIGURE 5 | Group means $(N=13)$ of mean normalized N1m source strengths elicited by TS_1, TS_2, and TS_3 in both hemispheres with error bars denoting $95 \%$ confidence intervals. Black, gray, and white bars denote the "Identical," "Category," and "Distinct" sequences, respectively. [ ${ }^{\star} p<0.05,{ }^{\star \star} p<0.01$ (Bonferroni-corrected)].

$p=0.11$; “Identical_Left_TS_3": $p=0.51$; "Identical_ Right_TS_1": $p=0.97$; “Identical_Right_TS_2": $p=0.43$; "Identical_Right_TS_3": $p=0.35$; "Category_Left_TS_1": $p=0.57$; "Category_Left_TS_2": $p=0.42$; "Category_ Left_TS_3": $p=0.91$; “Category_Right_TS_1": $p=0.86$; "Category_Right_TS_2": $p=0.28$; “Category_Right_TS_3": $p=0.95$; “Distinct_Left_TS_1": $p=0.62$; "Distinct_Left_
TS_2": $p=0.33$; “Distinct_Left_TS_3": $p=0.78$; "Distinct_ Right_TS_1": $p=0.54$; “Distinct_Right_TS_2": $p=0.28$; "Distinct_Right_TS_3": $p=0.68)$. The three-way repeatedmeasures ANOVA applied to N1m latencies revealed neither a significant main effect [Sequence: $F_{(2.24)}=1.97, p=0.16$; Hemisphere: $F_{(1,12)}=0.01, p=0.93$; Position: $F_{(2,24)}=2.05$, $p=0.15$ ] nor a significant interaction between them. N1m 

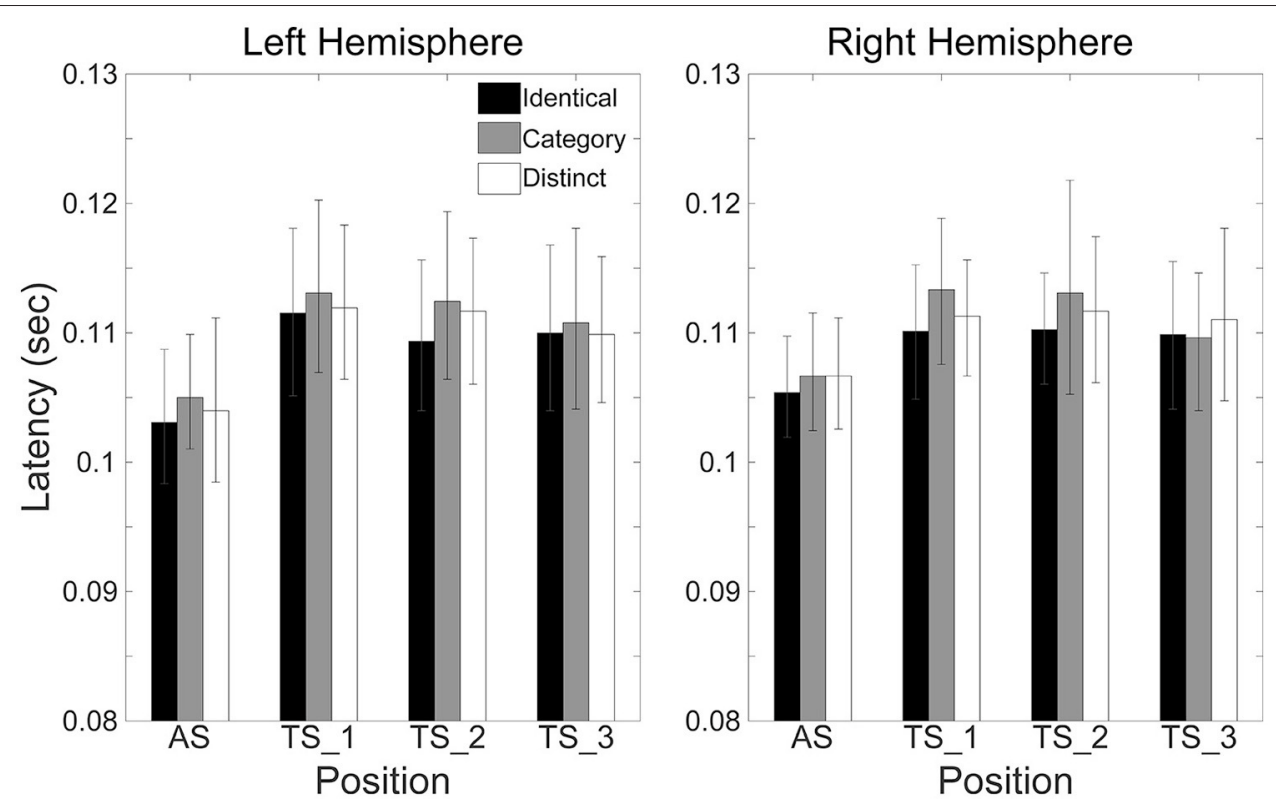

FIGURE 6 | Group means ( $N=13$ ) of N1m latencies in the left (left panel) and right (right panel) hemispheres elicited by the 1st (AS), 2nd (TS_1), 3rd (TS_2), and 4th (TS_3) trains of four successive FM sweeps, including error bars denoting $95 \%$ confidence intervals. Black, gray, and white bars denote the "Identical," "Category," and "Distinct" sequences, respectively.

latencies did not significantly differ between hemispheres or sequences.

\section{DISCUSSION}

In the present study, we investigated auditory evoked N1m responses elicited by four successive, temporally repeated, and superimposed FM sweeps by means of MEG. The results obtained demonstrated that the N1m source strengths elicited by TS were significantly influenced by the sequence of the FM sweep presentation. In contrast to our hypothesis, the successive presentation of four identical FM sweeps ("Identical" sequence) resulted in maximal normalized N1m source strengths, whereas those in the "Distinct" sequence in which four FM sweeps differed both in the FM modulation rate and/or direction and in carrier frequencies elicited minimal normalized N1m source strengths (Figures 4, 5). Moreover, the normalized N1m source strengths elicited in the "Category" sequence in which the FM sweeps had the same FM modulation rate and direction, but different carrier frequencies were around the middle of those elicited in the "Identical" and "Distinct" sequences.

The amplitude and latency of the N1m response is known to be influenced by the spectral components of the test sound signals (Pantev and Lutkenhöner, 2000; Roberts et al., 2000); however, in the present study, we used temporally repeated and superimposed FM sweeps that were balanced with respect to the spectral components (Figure 1). Moreover, we prepared 48 FM sweeps for each sound type (FM_up_04, FM_up_16, FM_down_04, or FM_down_16), resulting in 192 FM sweeps. We presented them once in each position (AS, TS_1, TS_2, or TS_3) in each sequence ("Identical," "Category," or "Distinct"). Therefore, the total bottom-up sound inputs were identical between sequences; only the sound presentation patterns differed between sequences ("Identical," "Category," or "Distinct"), and thus, may have had a significant impact on N1m responses. The results obtained suggest that neural adaptation in the human auditory cortex is sensitive to the rate and direction of FM sweeps and their carrier frequencies.

The results obtained appear to be contradictory to previous findings showing that the $\mathrm{N} 1 / \mathrm{N} 1 \mathrm{~m}$ responses elicited by repetitive identical pure tones were smaller than those elicited by successive distinct pure tones (Butler, 1968; Sams et al., 1985; Lagemann et al., 2012). Previous studies showed that neurons in the auditory cortex are sensitive to the rate and/or direction of FM sweeps in animals (Mendelson and Cynader, 1985; Heil and Scheich, 1992; Heil et al., 1992; Mendelson et al., 1993; Nelken and Versnel, 2000; Tian and Rauschecker, 2004; Godey et al., 2005; Atencio et al., 2007; Brown and Harrison, 2009; Trujillo et al., 2011) and humans (Hall et al., 2000, 2002; Hsieh et al., 2012; Joanisse and Desouza, 2014; Okamoto and Kakigi, 2015). The neural processing of repetitive FM sweeps in the human auditory cortex appears to differ from that of repetitive pure tones. Previous MEG studies using a two-tone adaptation paradigm supported our results by demonstrating that auditory evoked fields elicited by subsequent FM sweeps were larger when the preceding and subsequent FM sweeps were identical than when they had opposite FM directions; however, this effect was not observed in repetitive complex tones (Heinemann et al., 2010, 2011). These findings were consistent with our results demonstrating maximal normalized N1m source strengths elicited by four repetitive identical FM sweeps in the "Identical" sequence. Moreover, we found that normalized N1m source strengths in the "Category" sequence were significantly smaller than those in the "Identical" sequence. In the "Category" 
sequence, even though they had the same FM rate and direction, the four successive TS were characterized by different carrier frequencies, leading to different sensations in pitch and timber. Exposure to four successive sounds with the same pitch and timber may have resulted in larger $\mathrm{N} 1 \mathrm{~m}$ responses in the "Identical" sequence than in the "Category" sequence.

Inhibitory and excitatory neural circuits appear to contribute to neural adaptation in the auditory cortex (Whitmire and Stanley, 2016). Recent advances in genetic technology make it possible to activate and inactivate cell-type-specific neural circuits in behaving animals (Luo et al., 2008). Recent studies (Aizenberg et al., 2015; Natan et al., 2015) demonstrated that parvalbumin-positive interneurons inhibited neural responses to "standard" tones and "deviant" tones, whereas somatostatinpositive interneurons specifically reduced excitatory neural activity to "standard" tones. Moreover, parvalbumin-positive neurons and somatostatin-positive neurons appear to play major roles in fast responding inhibition and slow and long-lasting inhibition, respectively ( $\mathrm{Li}$ et al., 2014, 2015). This slower late frequency-specific inhibitory neural activity (0.2-0.4 s) may account for the adaptation of $\mathrm{N} 1 \mathrm{~m}$ responses elicited by repetitive pure tones. However, we herein used temporally repeated and superimposed FM sweeps as AS and TS, which were matched in the spectral domain, and changed their frequency components over time. Therefore, unlike repetitive pure tones, somatostatin-positive interneurons may not effectively inhibit the neural activity elicited by repetitive FM sweeps. Interactions between multiple excitatory-inhibitory neural circuits including parvalbumin-positive interneurons and somatostatin-positive interneurons may lead to the different sequence effects on N1m responses elicited by repetitive pure tones and FM sweeps.

In the present study, the auditory evoked $\mathrm{N} 1 \mathrm{~m}$ responses elicited by TS in all sequences ("Identical," "Category," and "Distinct") were smaller than those elicited by AS (Figures 2-4). This result appears to be contradictory to previous findings showing that the 2nd FM sweep elicited larger N1m responses than the 1st FM sweep (Heinemann et al., 2010). The main reason for this inconsistency appears to be the difference in interstimulus intervals between FM sweeps. We adopted a longer inter-stimulus interval $(0.5 \mathrm{~s})$ between sounds than that used in the previous study $(0.2 \mathrm{~s})$. Previous electroencephalography and MEG studies reported that inter-stimulus intervals shorter than $0.5 \mathrm{~s}$ may cause enhanced N1/ N1m responses (Budd and Michie, 1994; Loveless et al., 1996). Moreover, in the present study, we corrected the baseline relative to a 0.1 -s pre-stimulus interval for each position (1st, 2nd, 3rd, and 4th), whereas Heinemann et al. (2010) applied the baseline correction only once relative to a 0.1s pre-stimulus period before the 1st FM sweep. These differences in the experimental design appear to have led to differences in the results obtained.

The results of the present study showed that N1m source strengths elicited by AS and TS and normalized Nm source strengths elicited by TS were significantly larger in the right than in the left hemisphere. Moreover, a significant interaction between Sequence and Hemisphere in the present study indicated that neural modulation induced by successive FM sweeps might differ between hemispheres. The functional hemispheric asymmetries of the human auditory cortex are often observed in higher stage auditory processing. Previous neuroimaging studies revealed that the right hemisphere played an important role in listening to music (Zatorre et al., 1994; Griffiths et al., 1999), whereas the left hemisphere played a major role in speech processing (Eulitz et al., 1995; Alho et al., 1998; Belin et al., 2000; Szymanski et al., 2001). These functional hemispheric asymmetries may not be limited to complex natural sounds, but may originate from the neural processing of basic acoustic features. Positron emission tomography (Zatorre and Belin, 2001) and functional MRI (Jamison et al., 2006) studies demonstrated that the right hemisphere plays a dominant role in spectral processing. Previous MEG studies (Heinemann et al., 2011; Okamoto and Kakigi, 2015) also revealed that the N1m responses elicited by FM sweeps were larger in the right than in the left hemisphere. The right hemispheric dominance for FM sweep processing observed in the present study is also consistent with previous findings demonstrating that auditory cortex lesions in the right hemisphere caused severe impairments in detecting the frequency modulation of test sounds, whereas lesions in the left did not cause such an impairment in animals (Wetzel et al., 1998; Rybalko et al., 2006) or humans (Johnsrude et al., 2000).

In conclusion, using appropriately designed FM sweeps that were balanced between sequences and positions with respect to the total bottom-up sound inputs, we herein clearly demonstrated that the rate and/or direction of FM sweeps and their carrier frequencies influenced the $\mathrm{N} 1 \mathrm{~m}$ responses elicited by the successive FM sweeps. The results obtained suggest that the modulation of neural activity caused by successive FM sweeps differs from that of successive pure tones and may contribute to the efficient encoding of daily speech signals, which typically contain rapid repetitions of FM sweeps.

\section{AUTHOR CONTRIBUTIONS}

HO conceived and designed the study; HO performed experiments; $\mathrm{HO}$ analyzed data; $\mathrm{HO}$ interpreted results of experiments; $\mathrm{HO}$ prepared figures; $\mathrm{HO}$ drafted manuscript; $\mathrm{HO}$ and RK edited and revised manuscript; HO and RK approved final version of manuscript.

\section{FUNDING}

This work has been supported by the "Japan Society for the Promotion of Science for Young Scientists (26861426).”

\section{ACKNOWLEDGMENTS}

We are grateful to Yasuyuki Takeshima for his technical assistance and the participants for their diligent cooperation.

\section{SUPPLEMENTARY MATERIAL}

The Supplementary Material for this article can be found online at: http://journal.frontiersin.org/article/10.3389/fnhum. 2017.00036/full\#supplementary-material 


\section{REFERENCES}

Aizenberg, M., Mwilambwe-Tshilobo, L., Briguglio, J. J., Natan, R. G., and Geffen, M. N. (2015). Bidirectional regulation of innate and learned behaviors that rely on frequency discrimination by cortical inhibitory neurons. PLoS Biol. 13:e1002308. doi: 10.1371/journal.pbio.1002308

Alho, K., Connolly, J. F., Cheour, M., Lehtokoski, A., Huotilainen, M., Virtanen, J., et al. (1998). Hemispheric lateralization in preattentive processing of speech sounds. Neurosci. Lett. 258, 9-12. doi: 10.1016/S0304-3940(98)00836-2

Allison, T. (1962). Recovery functions of somatosensory evoked responses in man. Electroencephalog. Clin. Neurophysiol. 14, 331-343. doi: 10.1016/0013-4694(62)90110-4

Angel, R. W., Quick, W. M., Curtis Boylls, C., Weinrich, M., and Rodnitzky, R. L. (1985). Decrement of somatosensory evoked potentials during repetitive stimulation. Electroencephalogr. Clin. Neurophysiol. 60, 335-342. doi: 10.1016/0013-4694(85)90007-0

Atencio, C. A., Blake, D. T., Strata, F., Cheung, S. W., Merzenich, M. M., and Schreiner, C. E. (2007). Frequency-modulation encoding in the primary auditory cortex of the awake owl monkey. J. Neurophysiol. 98, 2182-2195. doi: 10.1152/jn.00394.2007

Belin, P., Zatorre, R. J., Lafaille, P., Ahad, P., and Pike, B. (2000). Voice-selective areas in human auditory cortex. Nature 403, 309-312. doi: 10.1038/35002078

Bregman, A. S. (1990). Auditory Scene Analysis: the Perceptual Organization of Sound. Cambridge, MA: MIT Press.

Brown, T. A., and Harrison, R. V. (2009). Responses of neurons in chinchilla auditory cortex to frequency-modulated tones. J. Neurophysiol. 101, 2017-2029. doi: 10.1152/jn.90931.2008

Buckner, R. L., Goodman, J., Burock, M., Rotte, M., Koutstaal, W., Schacter, D., et al. (1998). Functional-anatomic correlates of object priming in humans revealed by rapid presentation event-related fMRI. Neuron 20, 285-296. doi: 10.1016/S0896-6273(00)80456-0

Budd, T. W., Barry, R. J., Gordon, E., Rennie, C., and Michie, P. T. (1998). Decrement of the N1 auditory event-related potential with stimulus repetition: habituation vs. refractoriness. Int. J. Psychophysiol. 31, 51-68. doi: 10.1016/S0167-8760(98)00040-3

Budd, T. W., and Michie, P. T. (1994). Facilitation of the N1 peak of the auditory erp at short stimulus intervals. Neuroreport 5, 2513-2516. doi: 10.1097/00001756-199412000-00027

Butler, R. A. (1968). Effect of changes in stimulus frequency and intensity on habituation of the human vertex potential. J. Acoust. Soc. Am. 44, 945-950. doi: 10.1121/1.1911233

Chen, I. W., Helmchen, F., and Lütcke, H. (2015). Specific early and late oddballevoked responses in excitatory and inhibitory neurons of mouse auditory cortex. J. Neurosci. 35, 12560-12573. doi: 10.1523/jneurosci.2240-15.2015

Deboer, E., and Dreschler, W. (1987). Auditory psychophysics spectrotemporal representation of signals. Annu. Rev. Psychol. 38, 181-202. doi: 10.1146/annurev.ps.38.020187.001145

Eggermont, J. (1998). Representation of spectral and temporal sound features in three cortical fields of the cat. Similarities outweigh differences. J. Neurosci. 80, 2743-2764.

Eulitz, C., Diesch, E., Pantev, C., Hampson, S., and Elbert, T. (1995). Magnetic and electric brain activity evoked by the processing of tone and vowel stimuli. $J$. Neurosci. 15, 2748-2755.

Fruhstorfer, H., Soveri, P., and Järvilehto, T (1970). Short-term habituation of auditory evoked response in man. Electroencephalogr. Clin. Neurophysiol. 28, 153-161. doi: 10.1016/0013-4694(70)90183-5

Godey, B., Atencio, C. A., Bonham, B. H., Schreiner, C. E., and Cheung, S. W. (2005). Functional organization of squirrel monkey primary auditory cortex: responses to frequency-modulation sweeps. J. Neurophysiol. 94, 1299-1311. doi: 10.1152/jn.00950.2004

Griffiths, T. D., Johnsrude, I., Dean, J. L., and Green, G. G. (1999). A common neural substrate for the analysis of pitch and duration pattern in segmented sound? Neuroreport 10, 3825-3830. doi: 10.1097/00001756-199912160-00019

Hall, D. A., Haggard, M. P., Akeroyd, M. A., Summerfield, A. Q., Palmer, A. R., Elliott, M. R., et al. (2000). Modulation and task effects in auditory processing measured using fMRI. Hum. Brain Mapp. 10, 107-119. doi: 10.1002/1097-0193(200007)10:3<107::AID-HBM20>3.0.CO;2-8
Hall, D. A., Johnsrude, I. S., Haggard, M. P., Palmer, A. R., Akeroyd, M. A., and Summerfield, A. Q. (2002). Spectral and temporal processing in human auditory cortex. Cereb. Cortex 12, 140-149. doi: 10.1093/cercor/12.2.140

Heil, P., and Irvine, D. R. F. (1998). Functional specialization in auditory cortex: responses to frequency-modulated stimuli in the cat's posterior auditory field. J. Neurophysiol. 79, 3041-3059.

Heil, P., Rajan, R., and Irvine, D. R. F. (1992). Sensitivity of neurons in cat primary auditory cortex to tones and frequency-modulated stimuli. II: Organization of response properties along the 'isofrequency' dimension. Hear. Res. 63, 135-156. doi: 10.1016/0378-5955(92)90081-w

Heil, P., and Scheich, H. (1992). Spatial representation of frequency-modulated signals in the tonotopically organized auditory cortex analogue of the chick. $J$. Comp. Neurol. 322, 548-565. doi: 10.1002/cne.903220409

Heinemann, L. V., Kaiser, J., and Altmann, C. F. (2011). Auditory repetition enhancement at short interstimulus intervals for frequency-modulated tones. Brain Res. 1411, 65-75. doi: 10.1016/j.brainres.2011.07.009

Heinemann, L. V., Rahm, B., Kaiser, J., Gaese, B. H., and Altmann, C. F. (2010). Repetition enhancement for frequency-modulated but not unmodulated sounds: a human MEG study. PLoS ONE 5:e15548. doi: 10.1371/journal.pone.0015548

Hsieh, I. H., Fillmore, P., Rong, F., Hickok, G., and Saberi, K. (2012). FM-selective networks in human auditory cortex revealed using fMRI and multivariate pattern classification. J. Cogn. Neurosci. 24, 1896-1907. doi: 10.1162/jocn_a_00254

Jamison, H. L., Watkins, K. E., Bishop, D. V., and Matthews, P. M. (2006). Hemispheric specialization for processing auditory nonspeech stimuli. Cereb. Cortex 16, 1266-1275. doi: 10.1093/cercor/bhj068

Joanisse, M. F., and Desouza, D. D. (2014). Sensitivity of human auditory cortex to rapid frequency modulation revealed by multivariate representational similarity analysis. Front. Neurosci. 8:e306. doi: 10.3389/fnins.2014.00306

Johnsrude, I. S., Penhune, V. B., and Zatorre, R. J. (2000). Functional specificity in the right human auditory cortex for perceiving pitch direction. Brain 123, 155-163. doi: 10.1093/brain/123.1.155

Kourtzi, Z., and Kanwisher, N. (2000). Cortical regions involved in perceiving object shape. J. Neurosci. 20, 3310-3318.

Lagemann, L., Okamoto, H., Teismann, H., and Pantev, C. (2012). Involuntary monitoring of sound signals in noise is reflected in the human auditory evoked N1m response. PLoS ONE 7:e31634. doi: 10.1371/journal.pone.0031634

Li, L. Y., Ji, X. Y., Liang, F. X., Li, Y. T., Xiao, Z. J., Tao, H. Z. W., et al. (2014). A feedforward inhibitory circuit mediates lateral refinement of sensory representation in upper layer $2 / 3$ of mouse primary auditory cortex. J. Neurosci. 34, 13670-13683. doi: 10.1523/Jneurosci.1516-14.2014

Li, L. Y., Xiong, X. R. R., Ibrahim, L. A., Yuan, W., Tao, H. W. Z., and Zhang, L. I. (2015). Differential receptive field properties of parvalbumin and somatostatin inhibitory neurons in mouse auditory cortex. Cereb. Cortex 25, 1782-1791. doi: $10.1093 /$ cercor/bht 417

Liang, L., Lu, T., and Wang, X. Q. (2002). Neural representations of sinusoidal amplitude and frequency modulations in the primary auditory cortex of awake primates. J. Neurophysiol. 87, 2237-2261. doi: 10.1152/jn.00834. 2001

Liberman, A. M., Harris, K. S., Hoffman, H. S., and Griffith, B. C. (1957). The discrimination of speech sounds within and across phoneme boundaries. J. Exp. Psychol. 54, 358-368. doi: 10.1037/h0044417

Loveless, N., Levanen, S., Jousmaki, V., Sams, M., and Hari, R. (1996). Temporal integration in auditory sensory memory: neuromagnetic evidence. Electroencephalogr. Clin. Neurophysiol. 100, 220-228. doi: 10.1016/0168-5597(95)00271-5

Luo, L., Callaway, E. M., and Svoboda, K. (2008). Genetic dissection of neural circuits. Neuron 57, 634-660. doi: 10.1016/j.neuron.2008.01.002

Malmierca, M. S., Anderson, L. A., and Antunes, F. M. (2015). The cortical modulation of stimulus-specific adaptation in the auditory midbrain and thalamus: a potential neuronal correlate for predictive coding. Front. Syst. Neurosci. 9:e19. doi: 10.3389/fnsys.2015.00019

Malmierca, M. S., Sanchez-Vives, M. V., Escera, C., and Bendixen, A. (2014). Neuronal adaptation, novelty detection and regularity encoding in audition. Front. Syst. Neurosci. 8:e111. doi: 10.3389/fnsys.2014. 00111 
Mendelson, J. R., and Cynader, M. S. (1985). Sensitivity of cat primary auditory cortex (AI) neurons to the direction and rate of frequency modulation. Brain Res. 327, 331-335. doi: 10.1016/0006-8993(85)91530-6

Mendelson, J. R., Schreiner, C. E., Sutter, M. L., and Grasse, K. L. (1993). Functional topography of cat primary auditory cortex: responses to frequency-modulated sweeps Exp. Brain Res. 94, 65-87. doi: 10.1007/BF00230471

Näätänen, R., and Picton, T. (1987). The N1 wave of the human electric and magnetic response to sound: a review and an analysis of the component structure. Psychophysiology 24, 375-425. doi: 10.1111/j.1469-8986.1987.tb00311.x

Natan, R. G., Briguglio, J. J., Mwilambwe-Tshilobo, L., Jones, S. I., Aizenberg, M., Goldberg, E. M., et al. (2015). Complementary control of sensory adaptation by two types of cortical interneurons. Elife 4:e09868. doi: 10.7554/eLife.09868.001

Nelken, I., and Versnel, H. (2000). Responses to linear and logarithmic frequencymodulated sweeps in ferret primary auditory cortex. Eur. J. Neurosci. 12, 549-562. doi: 10.1046/j.1460-9568.2000.00935.x

Obleser, J., Eisner, F., and Kotz, S. A. (2008). Bilateral speech comprehension reflects differential sensitivity to spectral and temporal features. J. Neurosci. 28, 8116-8123. doi: 10.1523/JNEUROSCI.1290-08.2008

Okamoto, H., and Kakigi, R. (2015). Encoding of frequency-modulation (FM) rates in human auditory cortex. Sci. Rep. 5:e18143. doi: 10.1038/srep18143

Oldfield, R. C. (1971). The assessment and analysis of handedness: the Edinburgh inventory. Neuropsychologia 9, 97-113. doi: 10.1016/0028-3932(71)9 0067-4

Otsuru, N., Inui, K., Yamashiro, K., Urakawa, T., Keceli, S., and Kakigi, R. (2011). Effects of prior sustained tactile stimulation on the somatosensory response to the sudden change of intensity in humans: an magnetoencephalography study. Neuroscience 182, 115-124. doi: 10.1016/j.neuroscience.2011.03.019

Pantev, C., and Lutkenhoner, B. (2000). Magnetoencephalographic studies of functional organization and plasticity of the human auditory cortex. J. Clin. Neurophysiol. 17, 130-142. doi: 10.1016/0013-4694(94)00209-4

Reale, R. A., and Imig, T. J. (1980). Tonotopic organization in auditory cortex of the cat. J. Comp. Neurol. 192, 265-291. doi: 10.1002/cne.901920207

Roberts, T. P. L., Ferrari, P., Stufflebeam, S. M., and Poeppel, D. (2000). Latency of the auditory evoked neuromagnetic field components: stimulus dependence and insights toward perception. J. Clin. Neurophysiol. 17, 114-129. doi: 10.1097/00004691-200003000-00002

Robles, L., and Ruggero, M. A. (2001). Mechanics of the mammalian cochlea. Physiol. Rev. 81, 1305-1352.

Rugg, M. D., Soardi, M., and Doyle, M. C. (1995). Modulation of event-related potentials by the repetition of drawings of novel objects. Cogn. Brain Res. 3, 17-24. doi: 10.1016/0926-6410(95)00014-3

Rybalko, N., Suta, D., Nwabueze-Ogbo, F., and Syka, J. (2006). Effect of auditory cortex lesions on the discrimination of frequency-modulated tones in rats. Eur. J. Neurosci. 23, 1614-1622. doi: 10.1111/j.1460-9568.2006.04688.x

Sams, M., Paavilainen, P., Alho, K., and Naatanen, R. (1985). Auditory frequency discrimination and event-related potentials. Electroencephalogr. Clin. Neurophysiol. 62, 437-448. doi: 10.1016/0168-5597(85)90054-1

Schreiner, C. E., and Langner, G. (1988). Periodicity coding in the inferior colliculus of the cat. II. topographical organization. J. Neurophysiol. 60, $1823-1840$.
Szymanski, M. D., Perry, D. W., Gage, N. M., Rowley, H. A., Walker, J., Berger, M. S., et al. (2001). Magnetic source imaging of late evoked field responses to vowels: toward an assessment of hemispheric dominance for language. J. Neurosurg. 94, 445-453. doi: 10.3171/jns.2001.94.3.0445

Taaseh, N., Yaron, A., and Nelken, I. (2011). Stimulus-specific adaptation and deviance detection in the rat auditory cortex. PLOS ONE 6:e23369. doi: 10.1371/journal.pone.0023369

Tesche, C. D., Uusitalo, M. A., Ilmoniemi, R. J., Huotilainen, M., Kajola, M., and Salonen, O. (1995). Signal-space projections of MEG data characterize both distributed and well-localized neuronal sources. Electroencephalogr. Clin. Neurophysiol. Suppl. 95, 189-200. doi: 10.1016/0013-4694(95)00064-6

Tian, B., and Rauschecker, J. P. (1994). Processing of frequency-modulated sounds in the cats anterior auditory field. J. Neurophysiol. 71, 1959-1975.

Tian, B., and Rauschecker, J. P. (1998). Processing of frequency-modulated sounds in the cat's posterior auditory field. J. Neurophysiol. 79, 2629-2642.

Tian, B., and Rauschecker, J. P. (2004). Processing of frequency-modulated sounds in the lateral auditory belt cortex of the rhesus monkey. J. Neurophysiol. 92, 2993-3013. doi: 10.1152/jn.00472.2003

Trujillo, M., Measor, K., Carrasco, M. M., and Razak, K. A. (2011). Selectivity for the rate of frequency-modulated sweeps in the mouse auditory cortex. $J$. Neurophysiol. 106, 2825-2837. doi: 10.1152/jn.00480.2011

Uhlig, C. H., Dykstra, A. R., and Gutschalk, A. (2016). Functional magnetic resonance imaging confirms forward suppression for rapidly alternating sounds in human auditory cortex but not in the inferior colliculus. Hear. Res. 335, 25-32. doi: 10.1016/j.heares.2016.02.010

Ulanovsky, N., Las, L., and Nelken, I. (2003). Processing of low-probability sounds by cortical neurons. Nat. Neurosci. 6, 391-398. doi: 10.1038/nn1032

Wetzel, W., Ohl, F. W., Wagner, T., and Scheich, H. (1998). Right auditory cortex lesion in Mongolian gerbils impairs discrimination of rising and falling frequency-modulated tones. Neurosci. Lett. 252, 115-118. doi: 10.1016/S0304-3940(98)00561-8

Whitmire, C. J., and Stanley, G. B. (2016). Rapid sensory adaptation redux: a circuit perspective. Neuron 92, 298-315. doi: 10.1016/j.neuron.2016.09.046

Zatorre, R. J., and Belin, P. (2001). Spectral and temporal processing in human auditory cortex. Cereb. Cortex 11, 946-953. doi: 10.1093/cercor/11.10.946

Zatorre, R. J., Belin, P., and Penhune, V. B. (2002). Structure and function of auditory cortex: music and speech. Trends Cogn. Sci. (Regul. Ed). 6, 37-46. doi: 10.1016/S1364-6613(00)01816-7

Zatorre, R. J., Evans, A. C., and Meyer, E. (1994). Neural mechanisms underlying melodic perception and memory for pitch. J. Neurosci. 14, 1908-1919.

Conflict of Interest Statement: The authors declare that the research was conducted in the absence of any commercial or financial relationships that could be construed as a potential conflict of interest.

Copyright (C) 2017 Okamoto and Kakigi. This is an open-access article distributed under the terms of the Creative Commons Attribution License (CC BY). The use, distribution or reproduction in other forums is permitted, provided the original author(s) or licensor are credited and that the original publication in this journal is cited, in accordance with accepted academic practice. No use, distribution or reproduction is permitted which does not comply with these terms. 